\title{
CdSe Quantum Dots and SBMA/CdSe Nanocomposites Characterization by Optical and 2D DOSY NMR Methods
}

\author{
I.I. Geru" ${ }^{1, *}$, O.T. Bordian ${ }^{2}$, I.P. Culeac ${ }^{2}$, V.I. Verlan ${ }^{2}$ \\ ${ }^{1}$ Institute of Chemistry, Republic of Moldova \\ ${ }^{2}$ Institute of Applied Physics, Republic of Moldova
}

Copyright (C) 2015 by authors, all rights reserved. Authors agree that this article remains permanently open access under the terms of the Creative Commons Attribution License 4.0 International License

\begin{abstract}
We present experimental results on preparation and characterization of colloidal CdSe quantum dots (QDs) and polymer nanocomposite based on them. CdSe QDs were characterized by UV absorbance and visible photoluminescence (PL) spectroscopy as well as 2D DOSY NMR. The average CdSe particles size estimated from the UV-Vis absorption spectra was found to be in good correlation with results obtained from NMR measurements. Nanocomposite thin films were prepared on the base of styrene with butylmethacrilate copolymer (SBMA) (1:1). CdSe QDs were successfully incorporated into the SBMA copolymer matrix and thin films of CdSe/SBMA nanocomposites were characterized by UV-Vis and PL spectroscopy.
\end{abstract}

Keywords CdSe Nanocrystals, Quantum Dots, 2D DOSY NMR, Photoluminescence

\section{Introduction}

Colloidal luminescent CdSe quantum dots (QDs) remain the subject of great interest both for fundamental research and industrial development. Extensive research efforts have been developed over the last years on preparation and characterization of CdSe QDs because of their various applications in photonics, optoelectronics, medicine, etc., specifically for preparation of nanocomposite (NC) materials [1-3]. Application of CdSe QDs for preparation of polymer nanocomposites makes it possible to obtain materials with advanced properties for practical applications. In polymer nanocomposite the polymer is used both as a matrix, as well as passivation material for stabilization of $\mathrm{CdSe}$ nanoparticles properties.

The great advantage of QDs is the possibility they offer for tuning of their properties by varying the size of nanoparticles. The size of QDs can be easily controlled by different ways, for example by variation of the reaction time, or the temperature of the solvents, by variation of the concentration of the reaction compounds in solvents, etc. This gives a relatively simply and convenient technology for controlling the optical parameters of the nanocomposite structures for practical exploitation. For example, PL emission from colloidal CdSe quantum dots can be adjusted in a relatively wide spectral range. The technology makes it possible to prepare different sized nanocrystals with high PL efficiency and narrow PL band, while PL emission may cover a narrow spectral band, tuned from blue to read and even up to near infrared.

In this paper we present experimental results on preparation of colloidal CdSe QDs and their characterization by optical spectroscopy methods as well as by two-dimensional diffusion ordered NMR spectroscopy (2D DOSY NMR). The technology for synthesis of colloidal CdSe QDs was modified for preparation of SBMA copolymer nanocomposite based on them. The QDs have been incorporated into the copolymer matrix and the nanocomposite thin films were characterized by UV-Vis and PL spectroscopy. The mechanisms of PL emission and excited carries transport are discussed.

\section{Preparation Details}

\subsection{Materials}

Cadmium oxide (99.999\%), oleic acid (90\%), selenium (99.5\%, 100 mesh), trioctylphosphine (90\%), 1-octadecene $(90 \%)$, tributylphosphine $(90 \%)$, were purchased from Aldrich and used without further purification.

\subsection{Synthesis}

CdSe semiconductor nanocrystals were synthesized in a three-neck flask equipped with condenser, magnetic stirrer, thermocouple, and heating mantle. At the first step the stock solution was prepared: $180 \mathrm{mg}$ of Se powder, $3 \mathrm{~mL}$ of 
1-octadecene (ODE), $2 \mathrm{~mL}$ tributylphosphine (TBP) and 0.8 $\mathrm{mL}$ of trioctylphosphine (TOP) were mixed by intense stirring.

The Cd precursor solution was prepared from $80 \mathrm{mg}$ of CdO powder, $3 \mathrm{~mL}$ of 1 -octadecene, $1 \mathrm{~mL}$ of TBP and $4 \mathrm{~mL}$ of oleic acid. These compositions were mixed together by vigorous stirring. The precursor solution was heated up from the room temperature up to $\sim 220^{\circ} \mathrm{C}$ for complete dissolving of $\mathrm{CdO}$, which was determined visually. Then at the temperature $90^{\circ} \mathrm{C} 2 \mathrm{~mL}$ of the stock solution was quickly injected into the hot $\mathrm{Cd}$ precursor solution and then this mixture was kept at $90^{\circ} \mathrm{C}$. Different-sized QDs were obtained by varying the reaction time. For obtaining the desired size of quantum dots the colloidal quantum dots solutions were collected at determined reaction time intervals $\left(\sim 90^{\prime \prime}\right)$ after the injection. Extracted sample solutions were further introduced in chloroform solvent for preserving and further investigations. The CdSe QDs were isolated and purified by adding acetone to the cooled solutions followed by centrifugation at $8000 \mathrm{rpm}$ for about $30 \mathrm{~min}$.

In order to prepare the polymer nanocomposites, the SBMA copolymer and CdSe QDs were separately dissolved in toluene solvent, to obtain the solutions with the density $\rho=$ $111 \mathrm{mg} / \mathrm{mL}$ and $\rho=25 \mathrm{mg} / \mathrm{mL}$, respectively. These solutions were mixed together in the proportion $1: 1$ by vigorous stirring. The nanocomposites thin films were prepared on glass substrates by spin-coating method at $800 \mathrm{rpm}$ and dried out at $40^{\circ} \mathrm{C}$.

\section{Characterization Methods}

CdSe nanoparticles have been characterized by UV-Vis absorption and photoluminescent spectroscopy as well as by 2D diffusion ordered NMR spectroscopy. The average CdSe particles size for three selected samples was estimated from the position of the first excitonic peak in the UV-Vis absorption spectrum as well as from experimental data on 2D DOSY NMR. PL spectra were measured under excitation of a laser beam $405 \mathrm{~nm}$ using a MDR-23 monochromator and a photon counting module H9319-12 connected to a PC.

The principle of operation of diffusion ordered nuclear magnetic resonance spectroscopy is described in details in many sources [6-8]. It was demonstrated by NMR measurements that even after multiple centrifugations and washing of colloidal solution of $\mathrm{CdSe}$ nanoparticles in organic solvent, there is a remaining of molecular film of oleic acid on the surface of the nanoparticles. This is applied for determination of the nanoparticles size in the method of high resolution 2D DOSY NMR.

The method is based on separation of the NMR signals of different species according to their diffusion coefficients. It is well known that the character of Brownian motion of the molecules in solutions is determined not only by the temperature and viscosity, but also by the size and shape of these molecules.
In order to obtain the diffusion coefficients of the specific molecules a series of spin echo spectra is measured with different pulsed magnetic field gradient strengths. Subsequent processing of the signal decay gives information on the magnitude of diffusion coefficient. The initial diffusion weighted NMR spectra of DOSY NMR are one-dimensional, while 2D DOSY increases the dimensionality by one. In the case of high resolution $2 \mathrm{D}$ DOSY NMR relatively small difference in diffusion coefficient can be resolved [7,8]. Pulsed field gradient NMR spectroscopy can be used to measure translation diffusion of molecules. By use a gradient of magnetic field the molecules can be spatially labeled. If they move after this encoding during the diffusion time $(\Delta)$, their new position can be decoded by a second gradient. The measured signal is the integral over the whole sample volume, and NMR signal intensity is attenuated depending on the diffusion time and the gradient parameters $(\mathrm{g}, \delta)$ according to relation [8]:

$$
I=I_{\mathrm{o}} \exp \left(-D \gamma^{2} g^{2} \delta^{2}(\Delta-\delta / 3)\right),
$$

where $\mathrm{I}$ is the observed intensity; $\mathrm{I}_{0}$ is the reference intensity; $\mathrm{D}$ is the diffusion coefficient; $\gamma$ is the gyromagnetic ratio of the observed nucleus; $g$ is the gradient strength and $\delta$ is the length of the gradient.

Experimental measurements on 2D DOSY NMR were carried out with BRUKER Avance III NMR 400 spectrometer at room temperature at the following parameters of the system: gradient calibration $0.5566 \mathrm{G} / \mathrm{cm}$; $\mathrm{Z}$ gradient strength $5.57 \mathrm{G} / \mathrm{cmA}$; gradient pulse duration 1 $\mathrm{ms}$; delay for gradient recovery $0.1 \mathrm{~ms}$.

\section{Experimental Results and Discussion}

Fig. 1 illustrates the absorption spectra of colloidal chloroform solution of the prepared QDs for different particles size. The excitonic absorption peaks appear very clear on each curve, indicating the presence of CdSe QDs.

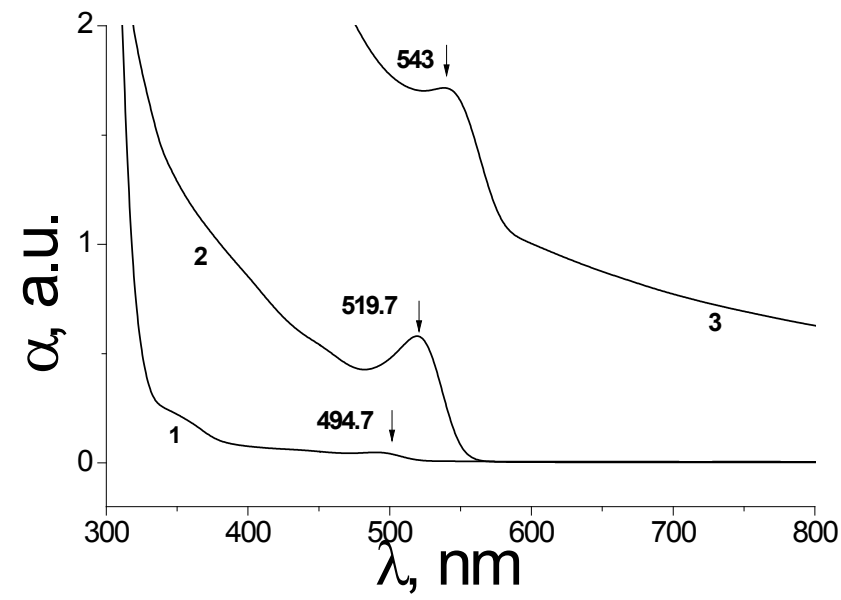

Figure 1. UV-Vis absorption spectra of suspension of CdSe quantum dots in chloroform grown at various reaction times (samples 1-3). The plots 1 - 3 correspond to three dots samples collected at $50 \mathrm{~s}$ time interval from the injection. The reaction times, $t$ : (1) $t_{0}$; (2) $t_{0}+50 \mathrm{~s}$; (3) $t_{0}+100 \mathrm{~s}$. 
The reaction time for selected samples 1,2, 3 increases by $50 \mathrm{~s}$, and the absorption peak red-shifts from 494.7 up to 543 $\mathrm{nm}$, sequentially. The absorption edge is relatively sharp, indicating a relatively narrow size distribution of the nanoparticles.

The size of QDs was estimated from the position of the lowest electron-hole transition (first exciton peak) in the absorption spectra ('Fig. 1). From the position of the first exciton peak the size of nanoparticles have been evaluated through the empirical relation [9]:

$$
d=\left(1.6122 \times 10^{-9}\right) \lambda^{4}-\left(2.6575 \times 10^{-6}\right) \lambda^{3}+\left(1.6242 \times 10^{-3}\right) \lambda^{2}-(0.4277) \lambda+41.57,
$$

where $\mathrm{d}(\mathrm{nm})$ is the average diameter of the CdSe QDs and $\lambda$ $(\mathrm{nm})$ is the wavelength of the first excitonic peak of the corresponding sample.

The size of CdSe quantum dots was varied for samples 1 3 by changing the reaction time, and was calculated as 2.28 , 2.42 and $2.92 \mathrm{~nm}$ respectively. This QDs size implies a strong confinement of the charge carriers, while the confinement energies of the electron and hole are larger than the Coulomb interaction [9-11]. In addition to this, the size of CdSe nanoparticles was calculated from the data obtained by 2D DOSY NMR. 'Fig. 2 illustrates 2D DOSY NMR display of a mixture of CdSe quantum dots, from the sample 3 and 2, dissolved in toluene and oleic acid. The diffusion axis F1 displays the numerical values of the logarithms of the diffusion coefficient $\mathrm{D}, \mathrm{m}^{2} / \mathrm{s}$ while the axis $\mathrm{F} 2$ displays the values of the absorption of ${ }^{1} \mathrm{H}$ NMR lines in ppm units. As a reference for absorption ( $0 \mathrm{ppm})$ dimethylsulfoxide was used. The spot at $6.4 \mathrm{ppm}, \log \mathrm{D}=-8.743 \mathrm{~m}^{2} / \mathrm{s}$ and $5.25 \mathrm{ppm}, \log \mathrm{D}$ $=-9.05 \mathrm{~m}^{2} / \mathrm{s}$ correspond to toluene molecule and free oleic acid molecule, while the spot at $5.7 \mathrm{ppm}, \log \mathrm{D}=-9.425 \mathrm{~m}^{2} / \mathrm{s}$ corresponds to protons of oleic acid on the surface of CdSe quantum dots. Using the standard processing procedure for 2D DOSY NMR processing data we obtained the average diameter of CdSe QDs for the sample 3 as $d_{3}=2.94 \mathrm{~nm}$. The average size of CdSe quantum dots of the samples 1 and 2 was obtained in a similar way. These data correlate well with the data obtained from the absorption spectra in UV-Vis (Table 1).

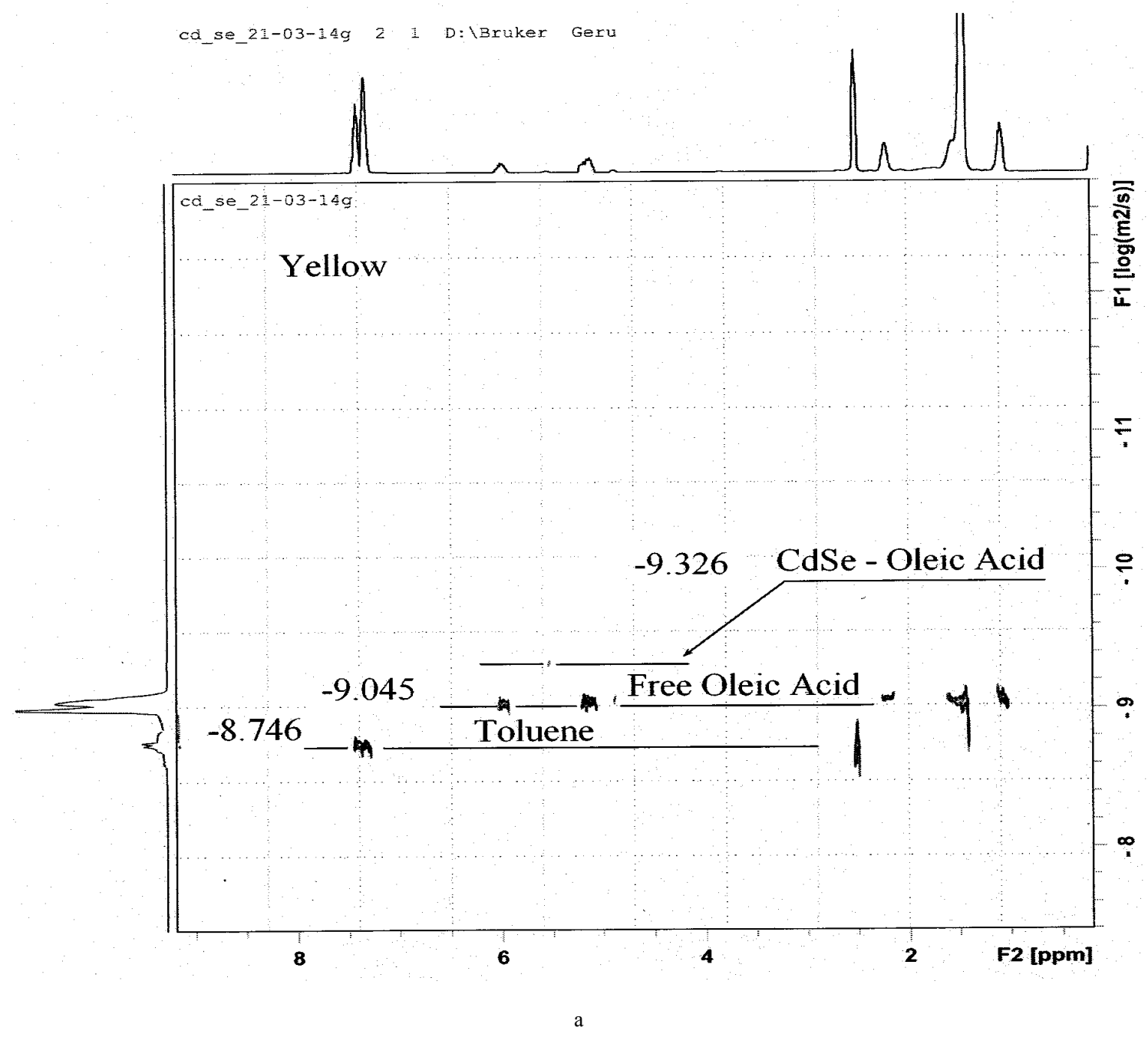




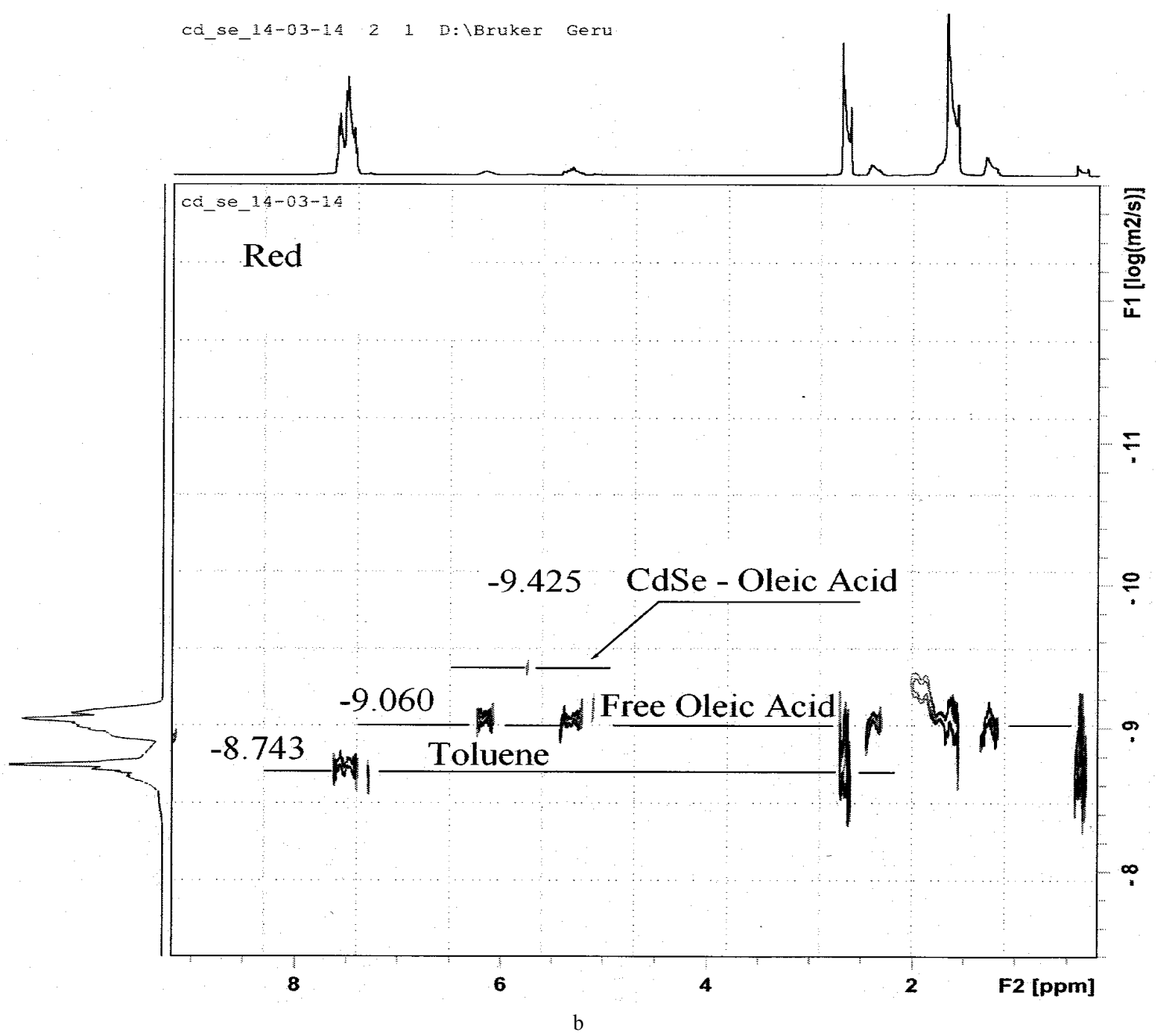

Figure 2. Illustration of 2D DOSY NMR spectra for a colloidal solution of CdSe QDs dissolved in toluene and oleic acid: (a) yellow emission sample (plot 2 in Fig. 1); (b) red emission sample (plot 3 in Fig. 1).

Table 1. The average sizes of CdSe quantum dots.

\begin{tabular}{|c|c|c|}
\hline Sample & $\begin{array}{c}\mathrm{d}, \mathrm{nm} \text { (From absorption } \\
\text { spectra) }\end{array}$ & $\begin{array}{c}\mathrm{d}, \mathrm{nm} \text { (From 2D } \\
\text { DOSY NMR) }\end{array}$ \\
\hline 1 & 2.28 & 2.32 \\
\hline 2 & 2.42 & 2.45 \\
\hline 3 & 2.92 & 2.94 \\
\hline
\end{tabular}

The energy of the main excitonic peak $\mathrm{E}$ as a function of the size of the nanocrystal is determined by the relation [12-14]:

$$
E=E_{g}+\frac{\hbar^{2} \pi^{2}}{2 R^{2}}\left(\frac{1}{m_{e}^{*}}+\frac{1}{m_{h}^{*}}\right)-\frac{1}{R}\left(\frac{1.8 e^{2}}{4 \pi \varepsilon_{0} \varepsilon_{r}}\right),
$$

where $E_{g}$ is the energy gap of bulk CdSe $\left(E_{g}=1.7 \mathrm{eV}\right), \mathrm{R}$ is the size of the nanoparticle, $\mathrm{m}_{\mathrm{e}}{ }^{*}$ and $\mathrm{m}_{\mathrm{h}}{ }^{*}$ are the effective masses of the electron and hole. The corresponding values for the effective masses are as reported elsewhere [12,13]: $\mathrm{m}_{\mathrm{e}} / \mathrm{m}_{0}=0.13, \mathrm{~m}_{\mathrm{h}} / \mathrm{m}_{0}=0.45$ and the dielectric constant $\varepsilon_{\mathrm{r}}=10.6$, $\varepsilon_{0}$ is the permittivity of free space and $e$ is the electron charge. If we take the particles diameter from the absorption spectra in 'Fig. 1 (sample 1-3) as $2.28 \mathrm{~nm}$ (1), $2.42 \mathrm{~nm}$ (2) and 2.92 $\mathrm{nm}(3)$ then the calculated exciton energies are respectively $2.31,2.24$ and $2.05 \mathrm{eV}$. The calculated values for the diameter of the nanocrystals are less than the bulk CdSe exciton Bohr radius $5.6 \mathrm{~nm}[9,10]$. Consequently, the quantum dots exhibit size-dependent properties [12, 13].

The exciton energy values obtained from 'Fig. 1 are higher than $\mathrm{E}_{\mathrm{g}}$ of the bulk CdSe $(1.7 \mathrm{eV}$ at $300 \mathrm{~K})$, which is an indicative of a blue shift of the absorption edge. The increase of the band gap is determined by the quantum size effect. Equation (2) illustrates the quantum confinement effect of quantum dots. In this equation the second term represents the quantum confinement term which is proportional to $\mathrm{R}^{-2}$, and shifts $\mathrm{E}$ to higher energy when the diameter of the nanoparticle decreases. The third term represents the Coulomb interaction, which is proportional to $\mathrm{R}^{-1}$. It shifts $\mathrm{E}$ to lower energy as the radius of the nanoparticles decreases. Consequently, when the particles size decreases the energy gap increases $[9,13]$.

The PL emission spectra of CdSe colloidal QDs solution in chloroform were registered at room temperature under the excitation of laser beam $405 \mathrm{~nm}$ ('Fig. 3). The PL spectra show a Stocks red-shift for each sample and the PL emission 
bands show relatively small FWHM values.

We can characterize the photoluminescence spectrum of CdSe quantum dots by Gaussian-shaped emission bands with the position of the peaks ranging from $508 \mathrm{~nm}$ to 566 $\mathrm{nm}$ depending on the size of CdSe quantum dots. The character of the photoluminescence spectra demonstrate that in the case CdSe quantum dots the PL mechanism is dominated by near band-edge emission. A relatively weak luminescence band in the read can be attributed to the emission processes associated with surface energy states. This luminescence band is strongly affected by technological parameters ('Fig. 3).

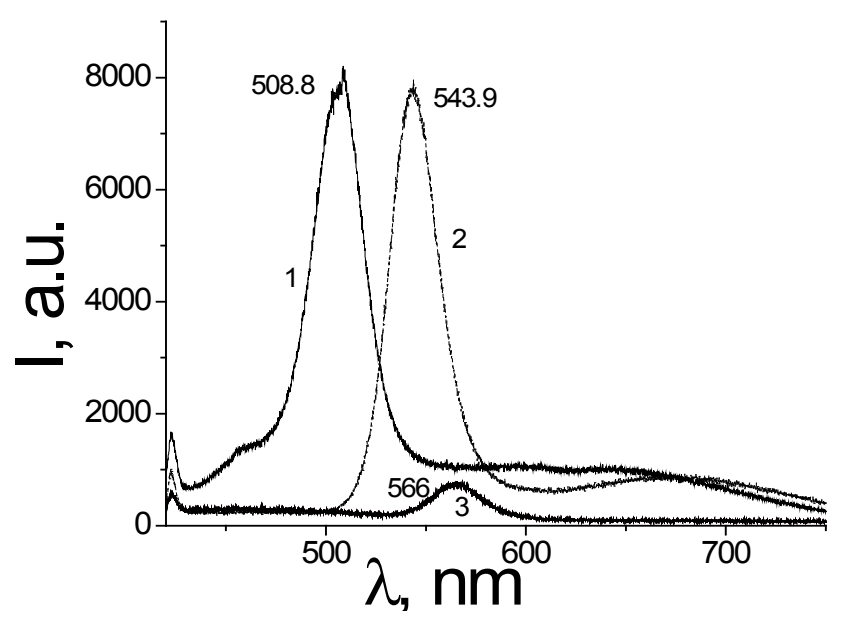

a

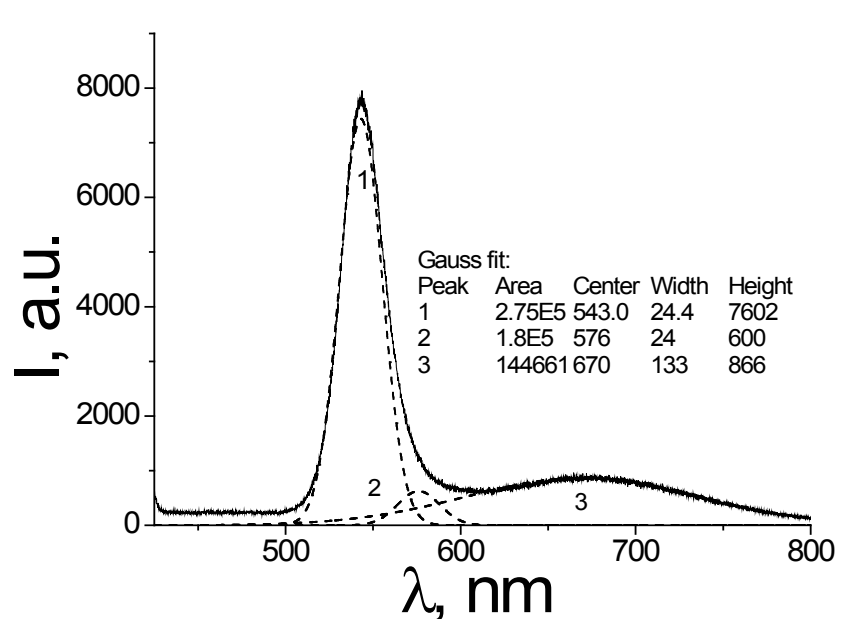

b

Figure 3. PL spectra of CdSe colloidal quantum dot samples: (a) PL spectra of CdSe colloidal quantum dot samples 1-3 dissolved in chloroform. The plots 1 - 3 correspond to three QDs samples, as indicated in 'Fig. 1. The excitation light is a laser beam $405 \mathrm{~nm}$. (b) Deconvolution of the PL spectrum for sample 2 .

The PL spectra can be decomposed by standard deconvolution method in elementary Gaussian lines ('Fig. 3b). For example, photoluminescence spectrum of the sample 2 was decomposed into a main PL band, positioned at $543 \mathrm{~nm}$ and two weak bands shifted to the red, at 576 and
$670 \mathrm{~nm}$. The FWHM of the main PL band at $543 \mathrm{~nm}$ is $\sim 24$ $\mathrm{nm}$. With increasing the reaction time, we observe an increasing of the diameter of CdSe nanoparticles; on the other hand, one can observe the shift of photoluminescence bands from 508 to $566 \mathrm{~nm}$. This correlates with the shift of the position of main absorption peak in the excitonic absorption spectrum ('Fig. 1). The relatively small value of FWHM of the photoluminescence bands suggests a small dispersion of the size of the CdSe quantum dots. The low intensity of the photoluminescence bands associated with the surface defects demonstrates a small density of the defects on the surface of quantum dots.

By selection of the technological parameters for the synthesis of $\mathrm{CdSe}$ quantum dots one can obtain quantum dots with desired PL characteristics, as illustrated in 'Fig. 4.

CdSe QDs were successfully incorporated into the copolymer matrix of SBMA and thin films of CdSe/SBMA nanocomposite were prepared. Styrene with butylmethacrilate was used as a polymer matrix as well as for stabilization of QDs properties. Nanocomposite thin films were obtained by deposition of the solution SBMA/CdSe on a glass substrate by spin-coating method and dried out at $40^{\circ} \mathrm{C}$. CdSe QDs with the size of $2.0 \mathrm{~nm}$ have been used for preparation of nanocomposite thin films. Optical microscope images of the $\mathrm{NC}$ thin films are illustrated in 'Fig. 5. While at low magnification the films appear smooth, at larger magnification $(1000 \times)$ one can observe micron-sized irregularities of the films.

Fig. 6 illustrates the absorption and PL spectra of CdSe QDs used in preparation of the nanocomposite, dissolved in a solution of chloroform for low concentration of QD. The first excitonic peak is positioned at $\sim 456 \mathrm{~nm}$, corresponding to energy of $2.73 \mathrm{eV}$. The calculated size of CdSe quantum dots is $2.0 \mathrm{~nm}$. The UV-Vis absorption spectrum of CdSe/SBMA nanocomposites in 'Fig. 7 shows a blue shift compared to the band-gap energy of bulk CdSe. A number of absorption excitonic peaks are observed, for example, at 2.73 and 3.17 $\mathrm{eV}$, while the major PL emission band is observed at $533 \mathrm{~nm}$.

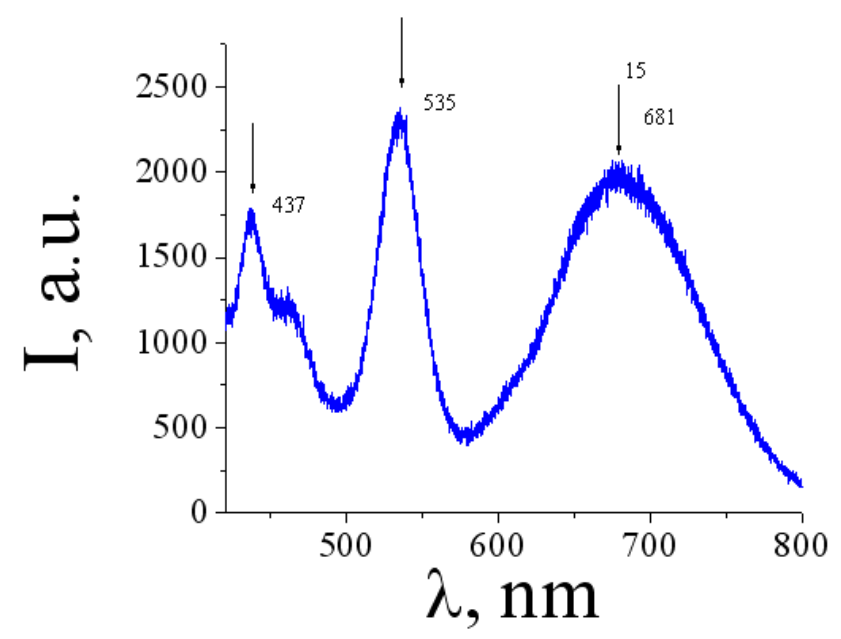




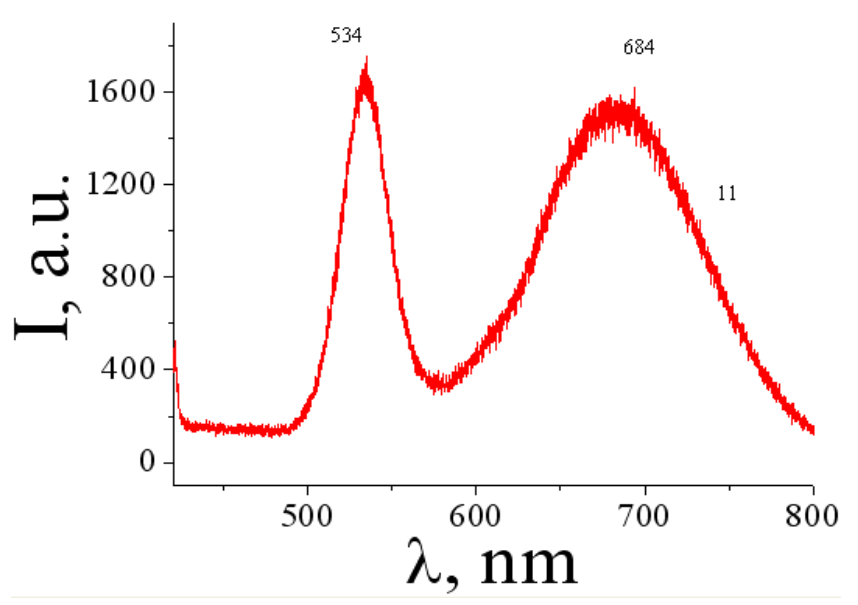

b

Figure 4. Variation of the PL emission spectrum of colloidal QDs by modification of the technological parameters: (a) - two PL bands (yellow and red); (b) - three PL bands (blue, green and red).

Fig. 8 illustrates the absorption spectra of the SBMA/CdSe nanocomposite films for higher concentration of CdSe quantum dots in the polymer matrix, $11.26 \%$ and $22.52 \%$. When comparing the absorption spectrum of the QDs with the same dimension and the corresponding spectrum of the $\mathrm{NC}$ we can see similar features. One can see more excitonic absorption bands that appear very clearly on each curve, indicating the presence of CdSe quantum dots. The position of the first exciton peak is registered at $452 \mathrm{~nm}$ and other excitons maximums are at 408, 390 and $371 \mathrm{~nm}$. The first exciton peak determines optical transitions under excitation $1 \mathrm{~S}_{3 / 2}(\mathrm{~h})-1 \mathrm{~S}(\mathrm{e})$. It is the same exciton transition as in the bulk material. The other excitons absorption peaks refer to other transitions of the confined QDs (Fig. 10). These transitions may be as following: $1 \mathrm{P}_{3 / 2}(\mathrm{~h})-1 \mathrm{P}(\mathrm{e})$ and $2 \mathrm{~S}_{3 / 2}(\mathrm{~h})-2 \mathrm{~S}(\mathrm{e})$. When increasing the concentration of QDs in the $\mathrm{NC}$ one can observe a slight increase of optical absorption of $\mathrm{NC}$ thin films, while the position of exciton absorption peaks does not vary with the concentration of QDs with the matrix itself.

The photoluminescence spectra of SBMA/CdSe nanocomposite films registered at room temperature are presented in 'Fig. 9. There is a substantial increase of the PL emission amplitude while increasing the concentration of QDs in the SBMA polymer matrix ( Fig. 9a). Deconvolution of the PL spectrum for $22.52 \%$ concentration of QDs is illustrated in 'Fig. 9b. The PL peak positions in deconvoluted spectrum are $457.5,525.7,602.7$, and $650.8 \mathrm{~nm}$. The half width of the main PL band at $543 \mathrm{~nm}$ is $\sim 55 \mathrm{~nm}$. We can see that the general character of PL spectra of the SBMA/CdSe nanocomposites and of CdSe quantum dots is a similar one. At the same time one can observe an increase in FWHM value. In the case of SBMA/CdSe nanocomposite it is about $55 \mathrm{~nm}$ compared to $\sim 24 \mathrm{~nm}$ in the case of CdSe quantum dots. The PL emission associated with QDs surface defect states has lower amplitude, in the case of nanocomposite, which can be determined by the passivation effect of the
SBMA polymer matrix. The Stokes shift for PL emission spectra is estimated as $\sim 73 \mathrm{~nm}$.

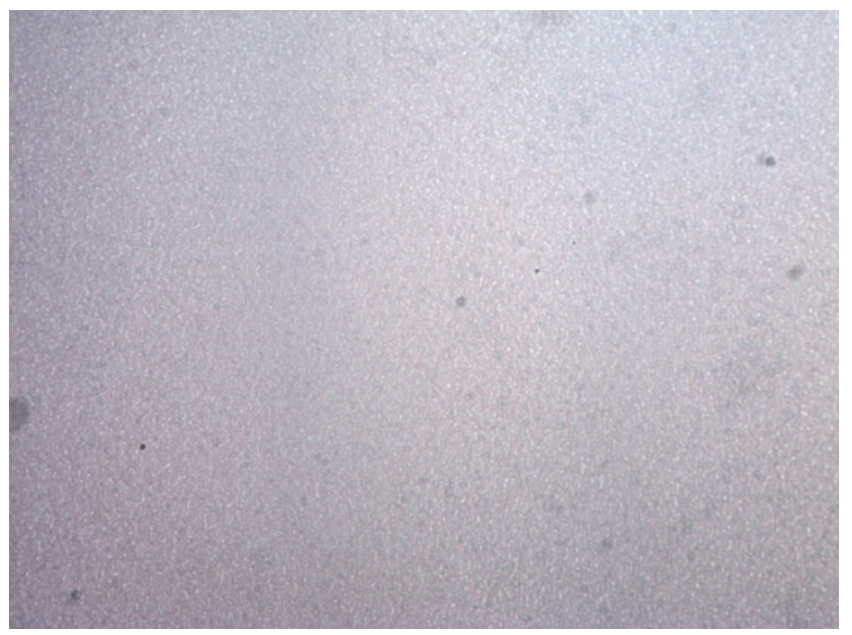

a

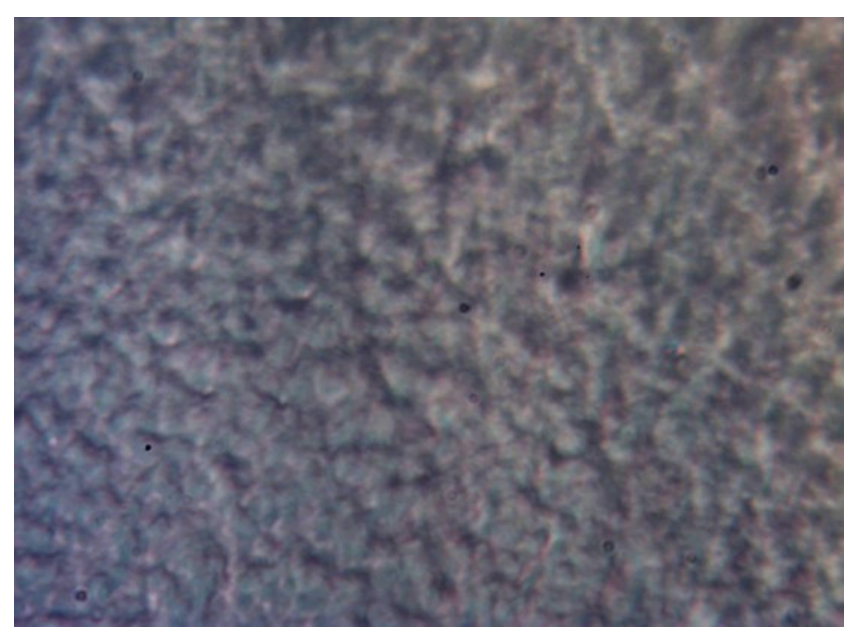

b

Figure 5. Reflection images of SBMA/CdSe nanocomposite thin films: (a) nanocomposite film at $100 \times$ magnification, the image width is $1.2 \mathrm{~mm}$; (b) Nanocomposite film at $1000 \times$ magnification, the image width is $127 \mu \mathrm{m}$. The concentration of CdSe QDs in the SBMA matrix is $22.52 \%$. The size of CdSe QDs used for preparation of $\mathrm{NC}$ thin films was $2.0 \mathrm{~nm}$.

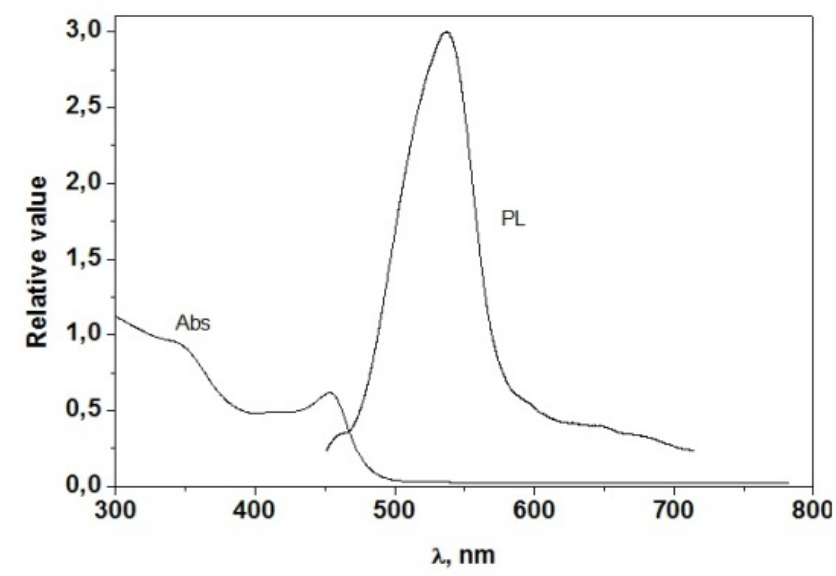

Figure 6. Illustration of absorption and PL spectra of CdSe QDs $(d=2.0$ $\mathrm{nm})$ in a solution of chloroform $(1.76 \%)$. 
We suppose that this is an indication that the quantum dots does not interact within the polymer matrix.

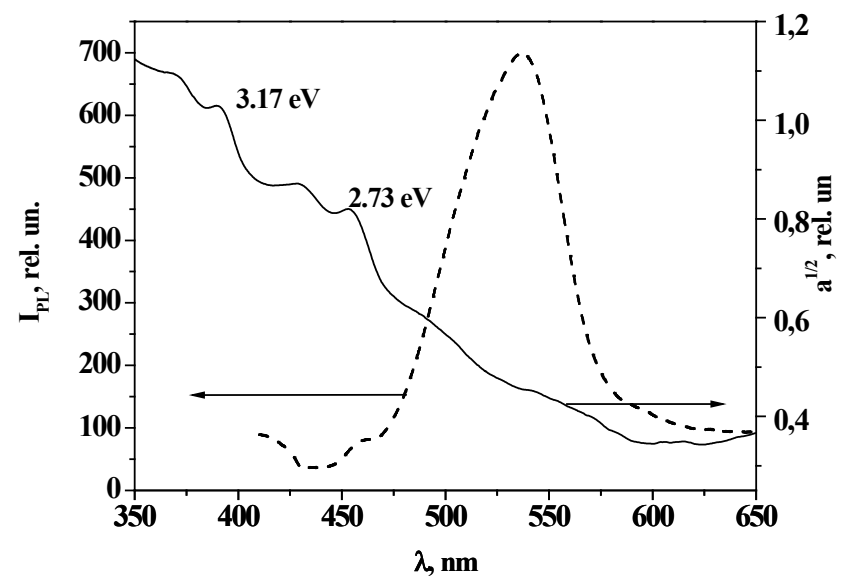

Figure 7. Illustration of absorption and PL spectra of CdSe QDs - SBMA nanocomposite for $1.76 \%$ concentration of QDs in $\mathrm{NC}(\mathrm{d}=2.0 \mathrm{~nm})$.

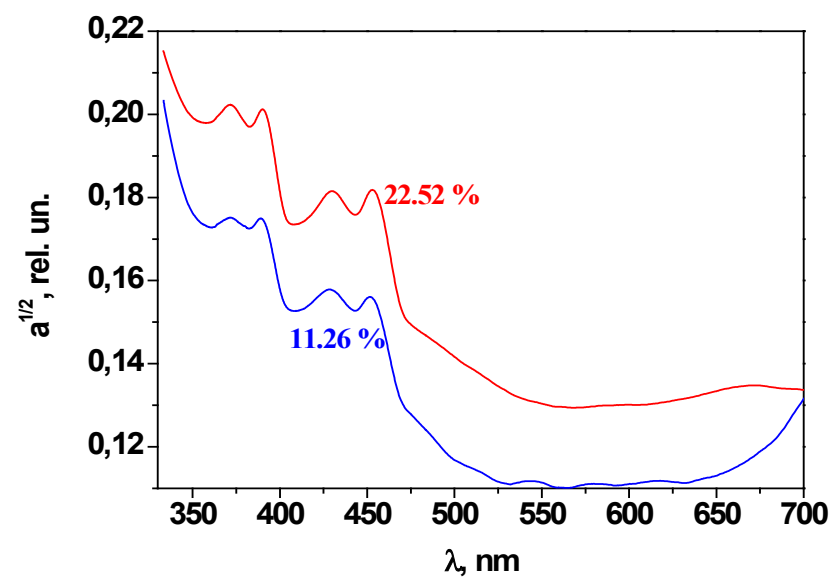

Figure 8. UV-Vis absorption spectra of the CdSe QDs - SBMA nanocomposite layers for two concentrations of CdSe quantum dots: $11.26 \%$ and $22.52 \%(\mathrm{~d}=2.0 \mathrm{~nm})$.

The QDs photoluminescence mechanism can be treated using the diagram in 'Fig. 10, which illustrates a simplified representation of the energy bands in the case of quantum dots versus the bulk band gap.
The size tuning properties of colloidal CdSe QDs are determined by the confinement of the exciton in a nanocrystal that is significantly smaller than the exciton in the bulk. This results in size-dependent optical properties. In theoretical interpretations this effect is described by a model of "quantum box" [2]. With decreasing the crystal size (radius) below the Bohr radius $\mathrm{R}_{B}\left(\mathrm{R}_{\mathrm{B}}\right.$ for CdSe is $\sim 6 \mathrm{~nm}$ ) the electrons and holes in the crystal are pressed into the "quantum box", and discrete energy levels similar as in the case of the atom appear. These states may be labeled as "atomic-like" with the appropriate notation s, p, d, etc., and all the related effects are referred to as quantum confinement effects.

For a spherical QD of radius $\mathrm{R}$ theoretical models [2,3] predict that the forbidden energy gap $E_{g}$ is simply proportional to $1 / \mathrm{R}^{2}$ (see eq. 2). Consequently, with decreasing the radius of QDs the band gap increases, that leads to modification of optical parameters. For a spherical QD of radius $\mathrm{R}$ theoretical models [2,3] predict that the forbidden energy gap $E_{g}$ is simply proportional to $1 / R^{2}$ (see eq. 2). Consequently, with decreasing the radius of QDs the band gap increases, that leads to modification of optical parameters. In addition to increasing $\mathrm{E}_{\mathrm{g}}$, the quantum confinement leads to a collapse of all continuous energy bands that are in the bulk material into discrete "atomic-like" energy levels. These well separated QD energy levels can be labeled using the atomic-like notations - 1S, 1P, etc., as shown in 'Fig. 10. The structure of discrete energy states result in specific features of absorption and luminescence spectra of quantum dots. Absorption spectrum of quantum dots bears the imprint of quantum dots energy levels.

The representation above illustrates the size dependent separation between the lowest electron $1 \mathrm{~S}(\mathrm{e})$ and hole $1 \mathrm{~S}(\mathrm{~h})$ QD states, obtained using the model "quantum box", which is characterized by discrete atomic like energy states. Lines and arrows indicate allowed well resolved interband optical transitions involving the states of electrons and holes $1 \mathrm{~S}$ and 1P. This fully characterizes the absorption spectrum of CdSe QD in NC with an average of $2.0 \mathrm{~nm}$. 

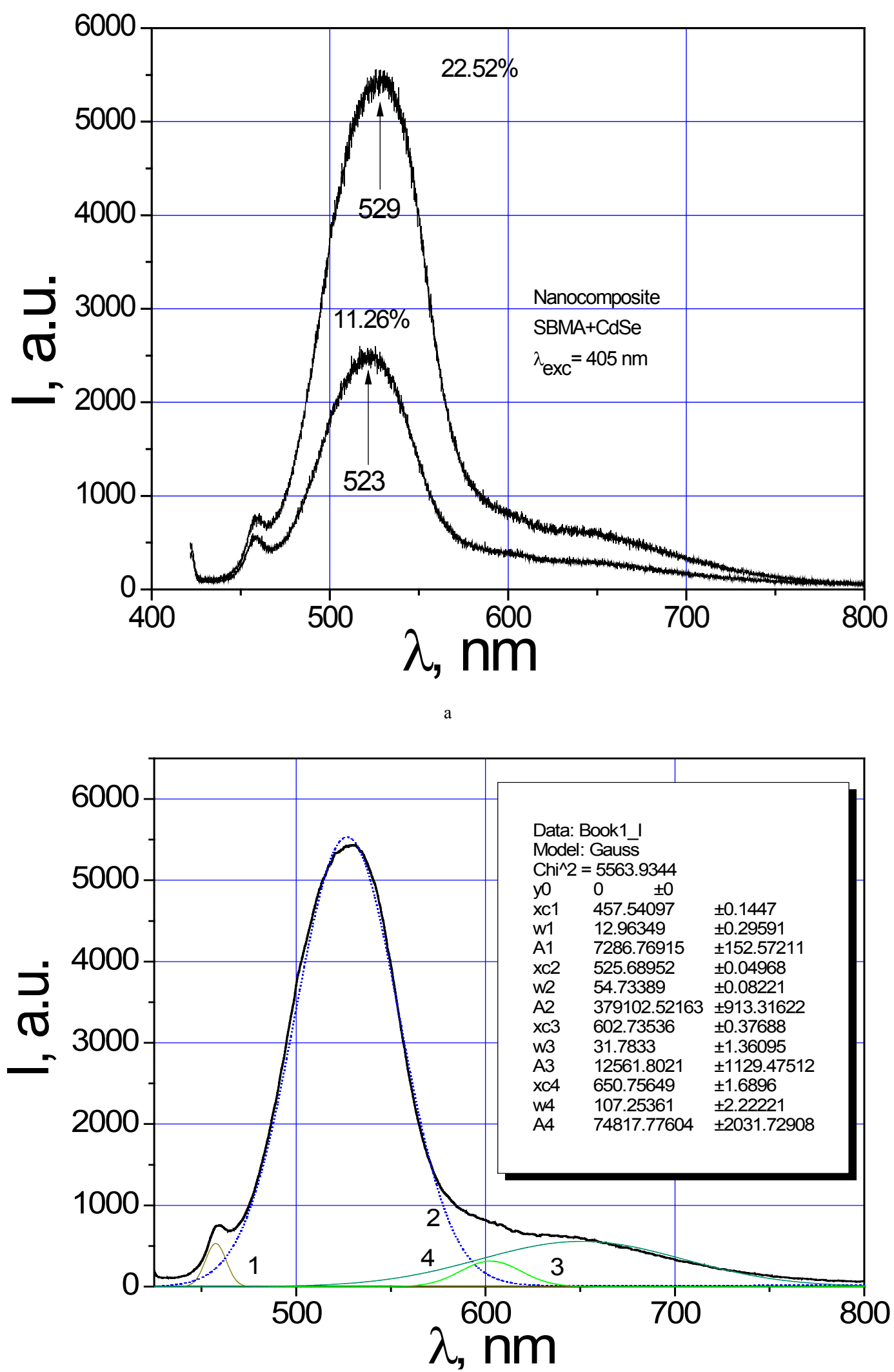

$\mathrm{b}$

Figure 9. (a) The photoluminescence spectra of SBMA/CdSe nanocomposite films for two concentrations of QDs in the polymer matrix: $11.26 \%$ and $22.52 \%$. The excitation source - $405 \mathrm{~nm}$ laser $(\mathrm{d}=2.0 \mathrm{~nm})$. (b) Deconvolution of the PL spectrum for $22.52 \%$ concentration of QDs. The PL peak positions are $457.5 ; 525.7 ; 602.7$; and $650.8 \mathrm{~nm}$. The half width of the main PL band at $543 \mathrm{~nm}$ is $55 \mathrm{~nm}$. 
Fig. 11 illustrates the mechanism of photoluminescence in CdSe QDs polymer nanocomposite. Because of the additional transfer of energy from the polymer matrix (Dexter and Forster transfer) $[14,15]$ to the CdSe QDs one can observe an enhancement of the PL emission in the polymer nanocomposite. The process of energy transfer is illustrated in 'Fig. 11 by bold arrows (Dexter transitions) and dotted (Forster) arrows.

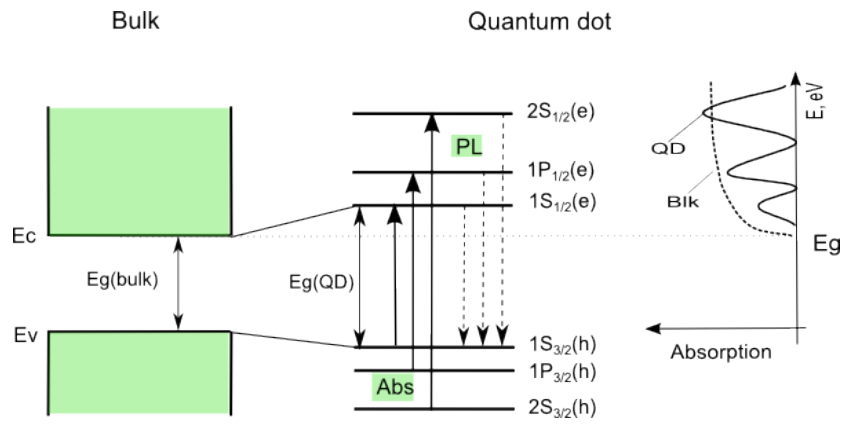

Figure 10. A simplified illustration of the energy bands in bulk material and CdSe quantum dots.

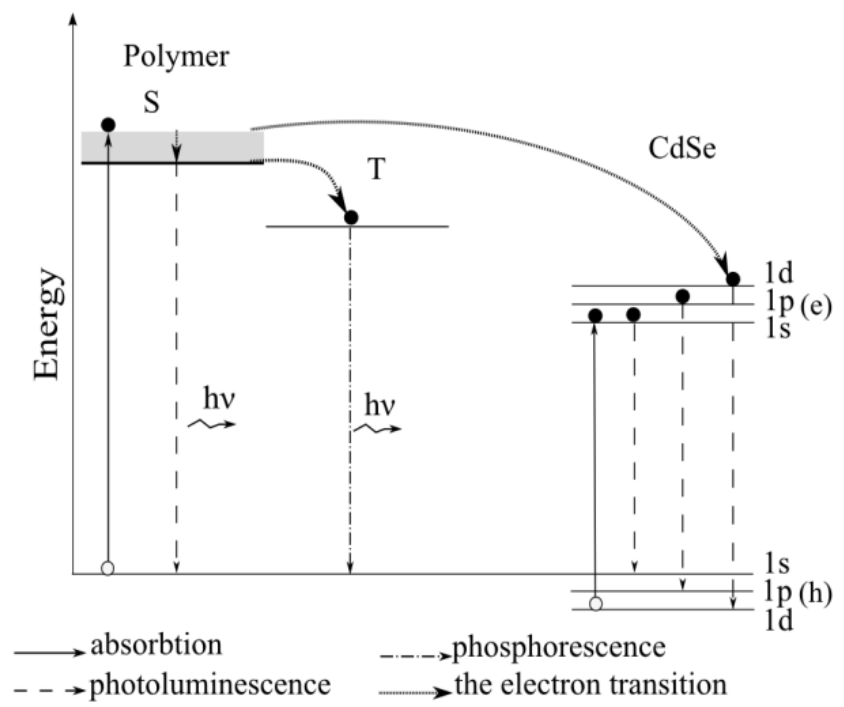

Figure 11. Illustration of the mechanism of photo-luminescence in CdSe QDs polymer nanocomposite.

\section{Conclusions}

We present experimental results on preparation and characterization of colloidal CdSe quantum dots (QDs) and polymer nanocomposite based on them. Nanocomposite thin films were prepared on the base of styrene with butylmethacrilate copolymer (SBMA). CdSe QDs were characterized by UV absorbance and visible photoluminescence (PL) spectroscopy as well as by $2 \mathrm{D}$ DOSY NMR. The average CdSe particles size estimated from the UV-Vis absorption spectra was found to be in good correlation with results obtained from NMR measurements. By adequate selection of technological parameters one can obtain CdSe QDs with desired PL emission bands, which can be applied for development of white light luminescent sources. We show the possibility for preparation of polymer nanocomposite based on CdSe QDs and SBMA polymer with enhanced PL emission. The photoluminescence spectra of CdSe QDs and nanocomposite SBMA/CdSe bears a similar character. We expect that the nanocomposite SBMA/CdSe can be used for optoelectronic applications.

\section{REFERENCES}

[1] Somers, R.C.; Bawendi, M.G.; Nocera, D.G. CdSe nanocrystal based chem-/bio- sensors, Chem. Soc. Rev., 2007, 36, pp. 579-591.

[2] Jorge, P.; Martins, M.A.; Trindade, T.; Santos, J.-L.; Farahi, F. Optical Fiber Sensing Using Quantum Dots, Sensors, 2007, 7, pp. 3489-3534.

[3] Yu, W.W.; Qu, L.; Guo, W.; Peng, X. Experimental Determination of the Extinction Coefficient of CdTe, CdSe, and CdS Nanocrystals, Chem. Mater., 2003, 15, pp. 2854-2860.

[4] Wang, Y.; Herron, N. Nanometer-Sized Semiconductor Clusters: Materials Synthesis, Quantum Size Effects, and Photophysical Properties, J. Phys. Chem. 1991, 95, pp. 525-532.

[5] Brus, L. Quantum crystallites and nonlinear optics, Appl. Phys. A, 1991, 53(6), pp. 465-474.

[6] C.S. Johnson Jr., Diffusion ordered nuclear magnetic resonance spectroscopy: principles and applications, Progress in Nuclear Magnetic Resonance Spectroscopy 34 (1999) 203-256

[7] Yoav Shrot, Lucio Frydman, Single-scan 2D DOSY NMR spectroscopy, Journal of Magnetic Resonance 195 (2008) 226-231

[8] Bruker manual: DOSY and Diffusion by NMR, 2002-2006, Bruker BioSpin GmbH, Rheinsfetten, Germany (http://www.nmr.ucdavis.edu/docs/dosy.pdf)

[9] Nguyen, H.Q. Synthesis and optical properties of CdSe nanocrystals and $\mathrm{CdSe} / \mathrm{ZnS}$ core/shell nanostructures in non-coordinating solvents, Adv. Nat. Sci.: Nanosci. Nanotechnology, 2010, 1, doi:10.1088/2043-6254/1/2/02500 4.

[10] Bawendi, M.G.; Steigerwald, M.L. and Brus, L.E. The Quantum Mechanics of Larger Semiconductor Clusters ("Quantum Dots"), Annu. Rev. Phys. Chem., 1990, 41, pp. 477-496.

[11] Biswas, A.and Ghosal, A. Hole Transport Characteristics of CdSe Single Quantum Wells at Low Temperatures, Journal of Electron Devices, 2011, 10, pp. 444-447.

[12] Meulenberg, R.W.; Lee, J.R.I.; Wolcott, A.; Zhang, J.Z.; Terminello L.J.; van Buuren, T. Determination of the Exciton Binding Energy in CdSe Quantum Dots, ACS Nano, 2009, 3 (2), pp. 325-330. 
[13] Jarosz, M. The physics and Chemistry of Transport in CdSe Quantum dot Solids, Ph.D. Thesis, Massachusetts Institute of Technology, USA, 2004.

[14] Helms Volkhard, Fluorescence Resonance Energy Transfer. Principles of Computational Cell Biology. Weinheim: Wiley-VCH. (2008), p. 202. ISBN 978-3-527-31555-0.
[15] Clifford B. Murphy, Yan Zhang, Thomas Troxler, Vivian Ferry, Justin J. Martin, and Wayne E. Jones, Jr. (2004). Probing Förster and Dexter Energy-Transfer Mechanisms in Fluorescent Conjugated Polymer Chemosensors. J. Phys. Chem. B, 108(5): p. 1537-1543, 004. doi:10.1021/jp0301406 